\title{
Conformational Transition Pathways in Major Facilitator Superfamily Transporters
}

\author{
Dylan Ogden, Kalyan Immadisetty, and Mahmoud Moradi* \\ Department of Chemistry and Biochemistry, University of Arkansas, Fayetteville, AR \\ 72701
}

E-mail: moradi@uark.edu 


\begin{abstract}
Major facilitator superfamily (MFS) of transporters consists of three classes of membrane transporters: symporters, uniporters, and antiporters. Despite such diverse functions, MFS transporters are believed to undergo similar conformational changes within their distinct transport cycles. While the similarities between conformational changes are noteworthy, the differences are also important since they could potentially explain the distinct functions of symporters, uniporters, and antiporters of MFS superfamily. We have performed a variety of equilibrium, non-equilibrium, biased, and unbiased allatom molecular dynamics (MD) simulations of bacterial proton-coupled oligopeptide transporter GkPOT, glucose transporter 1 (GluT1), and glycerol-3-phosphate transporter (GlpT) to compare the similarities and differences of the conformational dynamics of three different classes of transporters. Here we have simulated the apo protein in an explicit membrane environment. Our results suggest a very similar conformational transition involving interbundle salt-bridge formation/disruption coupled with the orientation changes of transmembrane (TM) helices, specifically H1/H7 and H5/H11, resulting in an alternation in the accessibility of water at the cyto- and periplasmic gates.
\end{abstract}




\section{Introduction}

Membrane transporters facilitate the exchange of materials across lipid bilayers. Among these transporters, the major facilitator superfamily (MFS) is the largest family of secondary membrane transporters, comprised by more than 10,000 members. ${ }^{1,2}$ Examples of MFS transporters that have been studied structurally include the lactose permease (LacY) from Escherichia coli (a suger-porter), ${ }^{3-5}$ the glycerol-3-phosphate transporter (GlpT), ${ }^{6} \mathrm{xy}-$ lose transporter(XylE), ${ }^{7}$ and the multidrug transporter EmrD. ${ }^{8}$

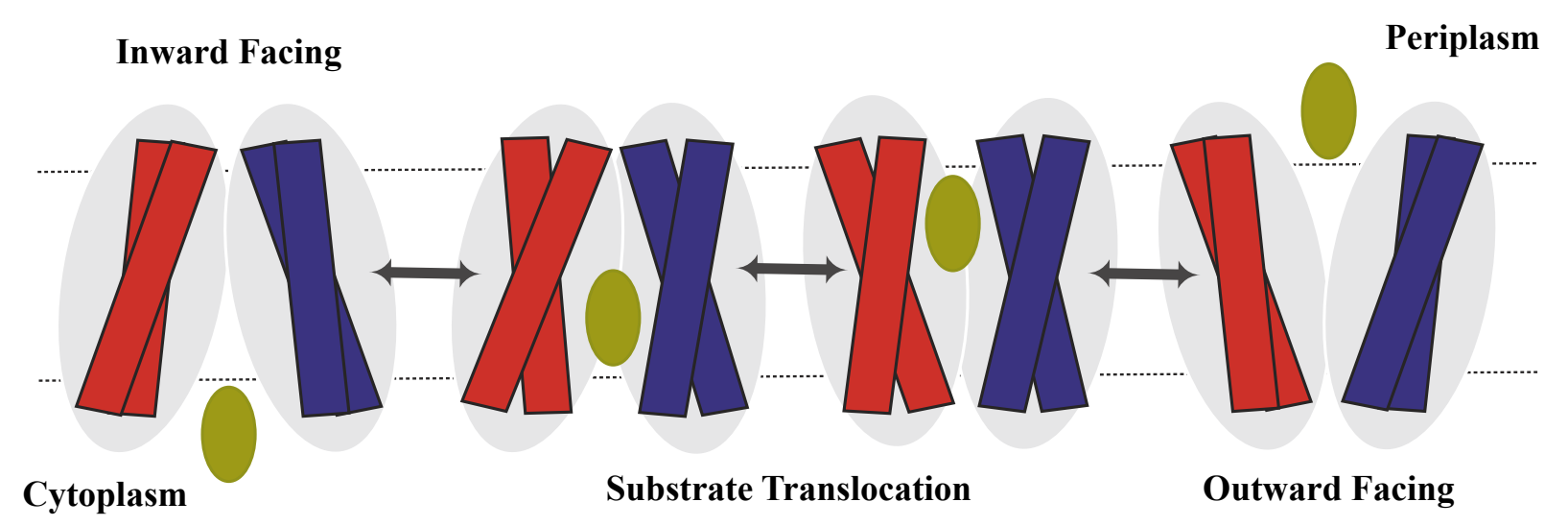

Figure 1: Schematic representation of the MFS facillitative diffusion of a substrate across cell membrane.

All MFS transporters share a common 12 TM helical structure, consisting of an N- and a C-bundle domain, displaying a twofold pseudo-symmetry. ${ }^{9}$ MFS transporters function in a number of different ways including active and passive transport. The latter function is uniport and the former is either symport or antiport. ${ }^{6,9,10}$ Despite these distinct functions, MFS transporters all share a common overall mode of function knwon as the "alternating access" mechanism, which is shared with other membrane transporters. ${ }^{1,9,10}$ According to this mechanism, the binding site is never exposed to both sides of membrane at the same time; instead, the protein alternates between an inward- (IF) and an outward-facing (OF) conformation. A more specific model of alternating access in MFS transporters has been proposed, which 
is known as the "rocker-switch" mechanism. ${ }^{6}$ In order for transport to ensue, the N- and Cbundle domains undergo concerted conformational changes, resembling a rocker-switch-like mode of movement that expose the binding site to the two sides of membrane alternatively and provide a mechanism for substrate translocation (Figure 1). In addition to the IF and OF states, the transport mechanism also involves occluded intermediates, where the binding site is exposed to neither side of the membrane.

Structural similarities of the MFS transporters suggest that these transporters may undergo similar conformational changes during their interconversions between the IF and OF states; although the coupling of these conformational changes to different binding/unbinding events is likely to be quite different in various symporters, uniporters, and antiporters. In order to study the similarities and differences of the structural changes in three different classes of MFS transporters, here we present a comparative view of IF-OF conformational changes of three proteins from three different classes of MFS transporters, including a bacterial proton-coupled oligopeptide transporter, namely GkPOT, ${ }^{11,12}$ the human glucose transporter 1 (GluT1), ${ }^{13,14}$ and the bacterial glycerol-3-phosphate transporter (GlpT). ${ }^{6}$ We specifically limit the discussion to the apo state of these proteins; however, we are aware that a full picture can only be provided if the full transport cycle is simulated. This includes the binding, unbinding, and translocation of substrates and their cotransported species. The study of apo protein IF-OF transition is the first step in characterizing the structural changes of MFS transporters within their transport cycle, which is the subject of the current study.

Proton-coupled oligopeptide transporters (POTs) are among the symporter members of the MFS superfamily. POTs uptake small peptides and peptide like molecules to the cell across the cell membrane using the inward directed proton electrochemical gradient as the source of energy for their active symport function. ${ }^{15,16}$ A very important feature among POTs is substrate promiscuity ${ }^{17}$ attributed to the binding site accommodating a range of peptides and peptide like molecules in multiple orientations. ${ }^{18}$ Mammalian POTs are yet to be crystallized; however, several bacterial POT members have recently been crystallized including 
PepT $_{s o},{ }^{18,19}$ PepT $_{s t},{ }^{11,20}$ PepT $_{s o 2},{ }^{12}$ and GkPOT. ${ }^{16}$ Particularly, the POT transporter from Geobacillus kaustophilus bacterium (GkPOT) has been crystallized in a lipidic environment to a remarkably high resolution of $1.9 \AA .{ }^{16} \mathrm{GluT} 1$, a uniporter belonging to the sugar porter subfamily of MFS transporters, mediates the uptake of glucose by passively transporting it along its concentration gradient. ${ }^{13}$ Although GluT1 is a passive transporter, recent structural studies suggest that the transport mechanism follows an alternating access mode of function similar to active membrane transporters. ${ }^{14,21,22}$ GlpT is an antiporter member of the MFS superfamily that uptakes glycerol-3-phosphate using the ouward directed inorganic phosphate concentration gradient as the source of energy for its active antiport activity. ${ }^{6,23}$ The binding of inorganic phosphate facilitates the transition from the IF to OF states, and the replacement of glycerol-3-phosphate reverts the protein back to the IF conformation. ${ }^{24,25}$

Molecular dynamics simulations have been a useful tool in the extensive study of proteins and membrane transporters such as GkPOT, ${ }^{26}$ GluT1, ${ }^{27}$ and GlpT. ${ }^{28}$ Unfortunately, due to the short timescales of typical unbiased molecular dynamics simulations, this technique is often incapable of capturing large-scale conformational changes. To be able to sample such conformations, enhanced sampling techniques are typically sought after, due to their ability to capture longer timescales. These include simulations such as steered molecular dynamics $(\mathrm{SMD})^{29}$ or targeted molecular dynamics (TMD). ${ }^{30}$ Admittedly, the transition between the IF and OF conformation may be sampled using unbiased MD for certain proteins. Recent work has shown a complete transition of GluT1 in 1-1.5 $\mu \mathrm{s} .{ }^{27}$ For slower IF-OF transitions, we have recently proposed a computational recipe that allows for reconstructing the IF-OF transition of various timescales. ${ }^{28,31,32}$ We have recently improved this protocol by employing a Riemannian description of conformational landscape of protein. ${ }^{33}$ The conformational transition pathways of GlpT has already been reported previously for the apo and phosphate-bound proteins. ${ }^{28}$ Here we compare the IF-OF conformational transition pathway of apo GkPOT and GluT1 to that of GlpT and discuss the similarities and differences of these pathways in detail. 


\section{Theoretical Methods}

Characterizing structural transitions of membrane transporters without compromising the detailed chemical description of these systems and their environments is computationally challenging mainly due to the prohibitively long timescales involved in such processes. Here we have employed all-atom MD to study the IF-OF conformational transitions of MFS transporters in the apo state. The GlpT simulations were previously reported ${ }^{28}$ and they are only included here for comparison. We were able to capture the GluT1 IF $\rightarrow$ OF transition using unibiased equilibrium MD; however, the $\mathrm{GkPOT} \mathrm{IF} \rightarrow \mathrm{OF}$ transition was not captured using such simulations. ${ }^{26}$ We therefore used the Riemannian ${ }^{33}$ version of the protocols developed for enhanced sampling simulations of $\mathrm{GlpT}^{28}$ to capture the $\mathrm{IF} \rightarrow \mathrm{OF}$ transition in аро GkPOT.

In each case, we used a membrane-embedded model of the wild-types apo protein (GkPOT, ${ }^{26}$ GluT1, and GlpT ${ }^{28}$ ) in its respective IF state based on the available crystal structure of protein (PDB: 4IKV ${ }^{16}$ 4PYP, ${ }^{14}$ and 1PW4, ${ }^{25}$ for GkPOT, GluT1, and GlpT, respectively). GkPOT and GlpT were placed in the 1-Palmitoyl-2-oleoyl-sn-glycero-3-phosphoethanolamine (POPE) lipids All the crystal structure waters were removed. The protocols for generating these models have been previously reported for GlpT ${ }^{28}$ and GkPOT $^{26}$ in detail. For GluT1, we used CHARMM-GUI ${ }^{34,35}$ for building the simulation system. The mutated residues in GluT1 crystal stricture (PDB: 4PYP) ${ }^{14}$ were substituted by the wild-type amino acids and the wild-type GluT1 was placed in a lipid bilayer of 1-Palmitoyl-2-oleoyl-sn-glycero-3phosphocholine (POPC), solvated with TIP3P waters, ${ }^{36}$ ionized with $0.15 \mathrm{M} \mathrm{KCl}$, and placed in a box of $\approx 106 \times 105 \times 104 \AA^{3}$.

We used CHARMM36 all-atom additive force field to describe all molecules. ${ }^{37,38}$ All MD simulations were performed with NAMD 2.11. ${ }^{39}$ Prior to equilibrium runs, each system was energy minimized for 10,000 steps using conjugate gradient algorithm ${ }^{40}$ and relaxed using a $\sim 1$ ns multi-step restraining procedure (CHARMM-GUI's default procedure for membrane 
proteins ${ }^{34}$ ). This initial relaxation was performed in an NVT ensemble while all production runs were performed in an NPT ensemble. Simulations were carried out using a 2 fs timestep at $310 \mathrm{~K}$ using a Langevin integrator with a damping coefficient of $\gamma=0.5 \mathrm{ps}^{-1}$. The pressure was maintained at 1 atm using the Nosé-Hoover Langevin piston method. ${ }^{41,42}$ The smoothed cutoff distance for non-bonded interactions was set to $10-12 \AA$, and long-range electrostatic interactions were computed with the particle mesh Ewald (PME) method ${ }^{43}$ and periodic boundary conditions were used in all simulations. GluT1 was equilibrated for $1 \mu \mathrm{s}$, which was long enough to capture the transition of the IF state to an OF state.

For GkPOT, the starting point for the simulations presented here was the equilibrated model from the unbiased simulations reported previously for the apo GkPOT. ${ }^{26}$ We then performed SMD simulations using various biasing protocols, described in detail in Supoprting Information. Here we only present the results of the most successful protocol, which uses a biasing potential based on the orientation of TM helices $\mathrm{H} 1, \mathrm{H} 2, \mathrm{H} 7$, and $\mathrm{H} 8$ to induce the $\mathrm{IF} \rightarrow \mathrm{OF}$ transition of apo GkPOT. The simulation time for this SMD protocol was 100 ns. During this simulation, a harmonic restraint was imposed on the orientation quaternions of the aforementioned helices with a varying harmonic center starting from the orientation quaternions of the initial conformation of the SMD simulation and changing towards those of a target structure that was built based on a homology model of MFS protein LacY, whose crystal structure is in the OF state (PDB: 3O7Q). ${ }^{44}$ The biasing potential was $\frac{1}{2} k \Omega^{2}$, where $k=10,000 \mathrm{kcal} /\left(\mathrm{mol} \AA^{2}\right)$ is the harmonic constant and $\Omega$ is the geodesic distance between the current orientation quaternion and a nonlinear interpolation of the initial and target orientation quaternions based on the Riemannian geometry. The outcome of the SMD simulation was equilibrated again for approximately 200 ns to determine the stability of the generated OF state of GkPOT. Prior to this follow-up equilibrium simulation, however, the final harmonic restraint on orientation quaternions were released gradually within a 20-ns SMD simulation with a fixed center an a variable force constant from 10,000 to $0 \mathrm{kcal} /\left(\mathrm{mol} \AA^{2}\right)$. Following the SMD simulations, the IF-OF conformational transition pathway was further 
relaxed using a Riemannian implementation of string method with swarms of trajectories (SMwST). ${ }^{28,33}$ The collective variables used for the SMwST simulations were the orientation quaternions of the TM helices H1-H12. The force constant was 10,000 kcal/( $\left.\mathrm{mol}^{2} \AA^{2}\right), 50 \mathrm{im}-$ ages $\times 20$ copies were used for these SMwST simulations (1000 replicas), and the restraining and release stages were each 5 ps long, iterated 100 times. All trajectories were collected with a frequency of 2 to 20 ps but typically a lower frequency was used for the analysis, which was conduced using VMD. ${ }^{45}$ 


\section{Results and Discussion}
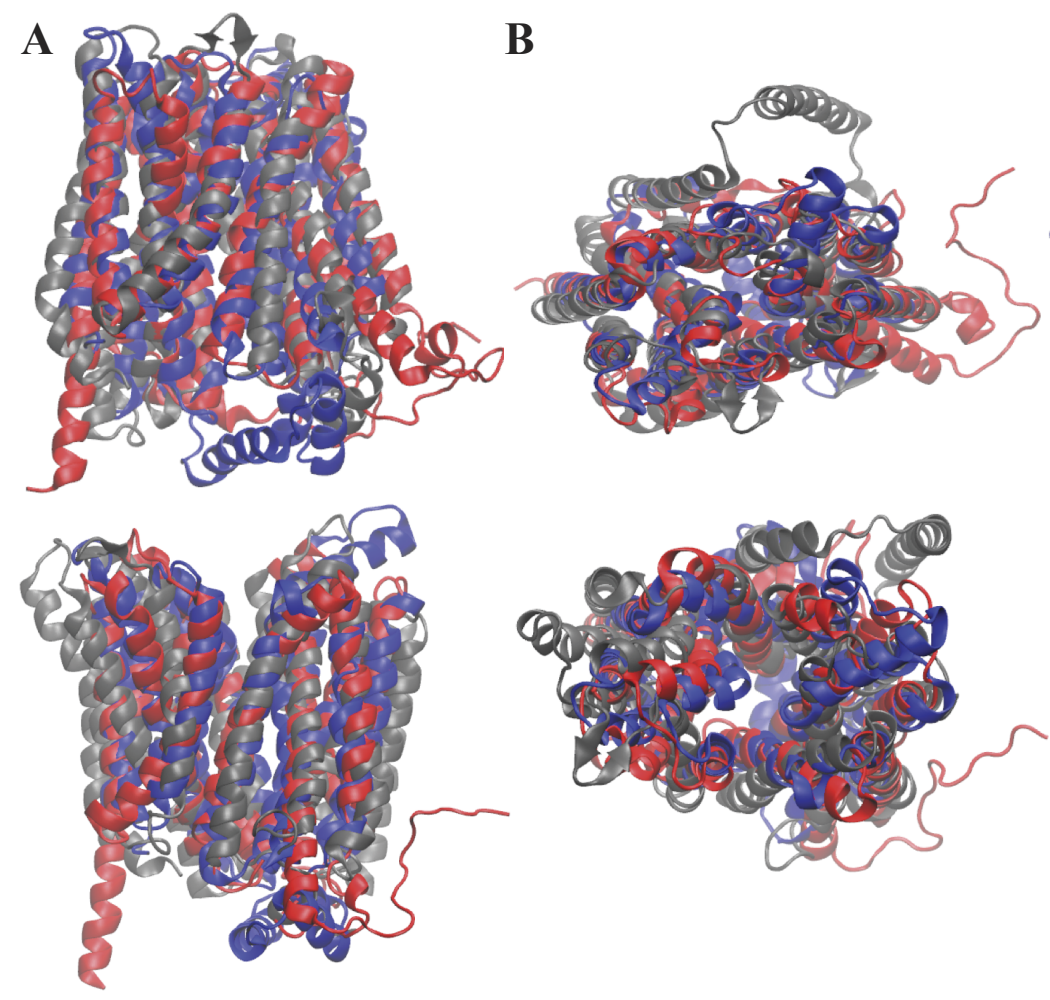

C
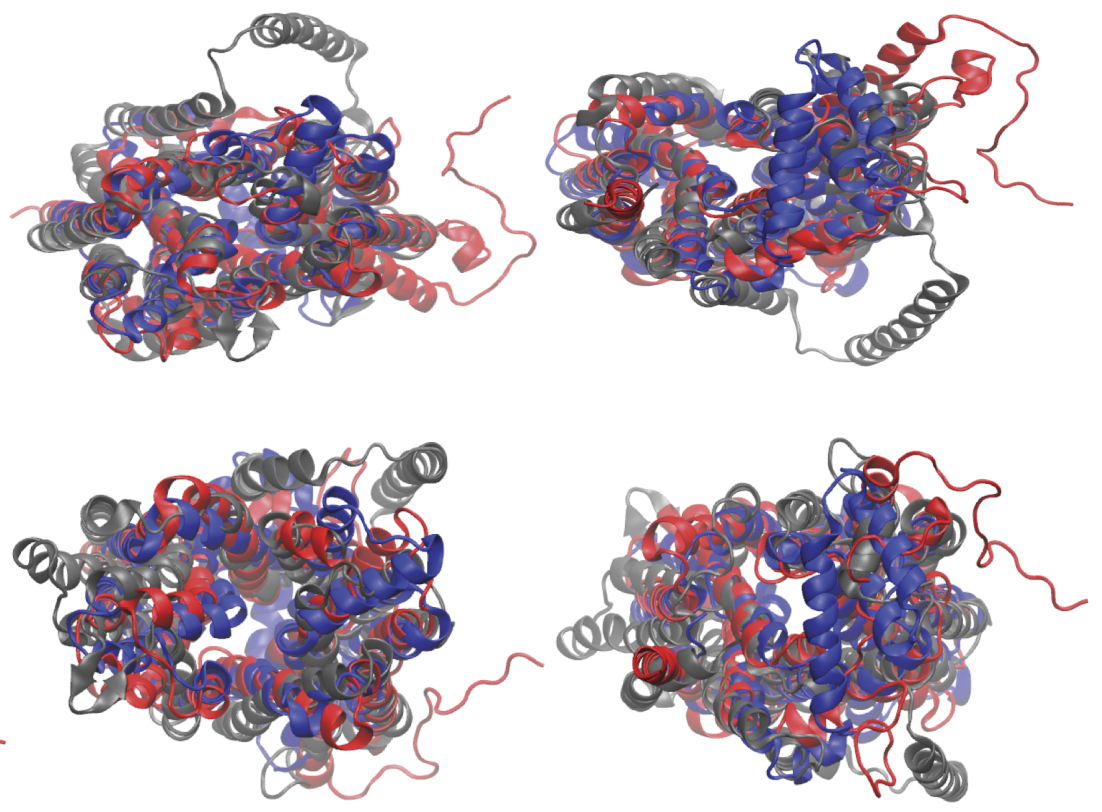

Figure 2: MFS trasnporters GkPOT (gray), and GluT1 (blue), and GlpT (red) in the IF (top) and OF (bottom) states from three different viewpoints including $(\mathrm{A})$ side, $(\mathrm{B})$ top, and $(\mathrm{C})$ bottom views.

The equilibrium simulation of wild-type GluT1 shows a transition from the IF state to an OF

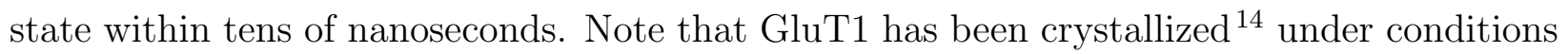
that are different from those used in this simulation. Specifically, N45T/E329Q mutant GluT1 has been used for crystallography, which is different from the wild-type GluT1 in our simulations. The fast transition is likely be attributed to the differences between the wildtype and N45T/E329Q mutant GluT1 proteins. However, here we assume that the transition observed in our simulation more or less reveals the important features of the IF-OF transition of apo GluT1.

Previously reported, ${ }^{26}$ 400ns simulations of GkPOT in various apo and substrate-bound 
conditions have revealed the shortcomings of unbiased MD for the study of global conformational changes in this protein. Therefore, the equilibrated structure of apo GkPOT was used here to initiate nonequilibrium pulling SMD simulations to capture the $\mathrm{IF} \rightarrow \mathrm{OF}$ conformational transition as described in Theoretical Methods. A number of nonequilibrium simulations were run to steer the IF GkPOT to the OF conformation. Of the combinations of transmembrane helices used to induce the transition, the combination of $\mathrm{H} 1, \mathrm{H} 2, \mathrm{H} 7$ and $\mathrm{H} 8$ provided the most successful attempt, evaluated by the stability of the resulting OF conformation; i.e., once equilibrated, the resulting conformation from the SMD simulation should stay in an OF conformation with an open periplasmic gate and a closed cytoplasmic gate. Similar to GkPOT, the simulations of GlpT were previously conducted using a similar biasing protocol, which only involved the TM helices H1 and H7. ${ }^{28}$ This protocol was also used in our GkPOT studies (see Supporting Information); however, the resulting OF conformation was not stable enough as compared to that generated using the $\mathrm{H} 1 / \mathrm{H} 2 / \mathrm{H} 7 / \mathrm{H} 8$ protocol.

\section{Structural Comparisons}

Structures of GkPOT, GlpT, and GluT1 are presented in Figure 2, depicting the IF and OF conformations of the respective proteins represented in the side, top, and bottom views. These proteins share a very similar topology, with the exception of (1) the intercoupling helix (ICH) present in GluT1 at the cytoplasmic gate and (2) the two additional TM helices (HA and $\mathrm{HB}$ ) in GkPOT. There are similarities and differences that are discussed more quantitatively below. 


\section{Global Protein Conformational Dynamics}
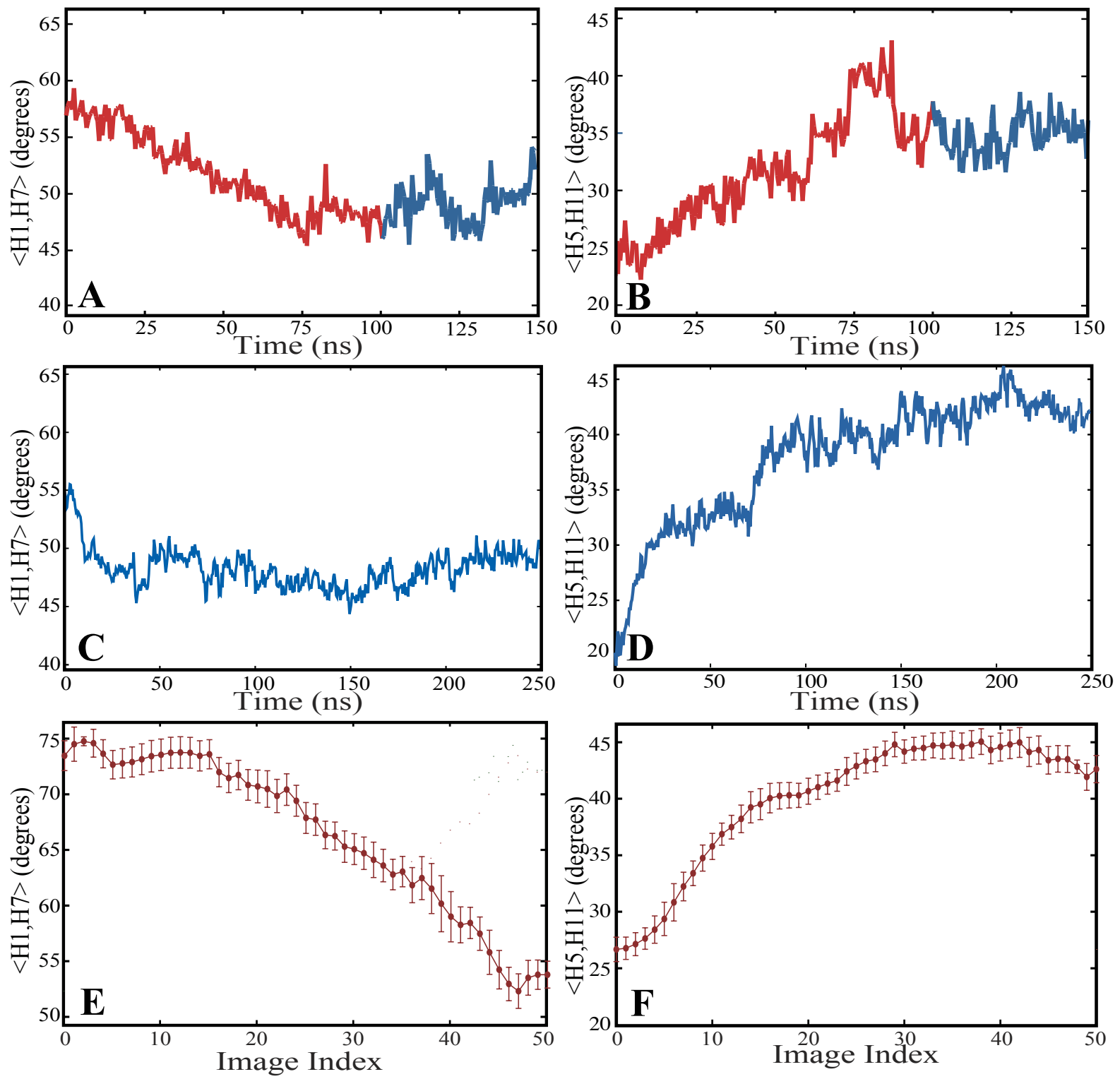

Figure 3: Changes in interhelical angles $¡ \mathrm{H} 1, \mathrm{H} 7 \AA$ and $¡ \mathrm{H} 5, \mathrm{H} 11$ in $\operatorname{GkPOT}(\mathrm{A}, \mathrm{B})$, GluT1 (C,D), and GlpT (E,F) simulations. GkPOT time series include SMD (red) and follow-up release (blue) simulations. GluT1 time series is based on unbiased equilibrium simulations. GlpT values are based on biase-exchange umbrella sampling simulations, where images 0 and 50 correspond to IF and OF states, respectively. ${ }^{28}$ 
Our previous simulations of GlpT revealed an important role for the interhelical angles between $\mathrm{H} 1$ and $\mathrm{H} 7(\langle\mathrm{H} 1, \mathrm{H} 7\rangle)$ and between H5 and H11 $(\langle\mathrm{H} 5, \mathrm{H} 11\rangle) .{ }^{28}$ The former is directly involved in periplasmic gating and the latter is involved in cytoplasmic gating. Figure 4 describes the changes associated with these interhelical angles for GkPOT, GluT1, and GlpT. The nature of the three sets of simulations are different; however, one can determine the trend of the changes in these interhelical angles in all three sets of simulations. with the SMD and follow-up release simulations described in Theoretical Methods, GkPOT transitions to a stable OF conformation. We have only shown initial part of the follow-up equilibrium simulations to make the comparison between the three transporters easier. See the Supporting Information for the time series associated with the full follow-up equilibrium simulations of GkPOT. To transition to OF the angle between $\mathrm{H} 1$ and $\mathrm{H} 7$ decreases from the cytoplamsic side while concurrently increases on the periplasmic side. Similarly, we are only showing the first $250 \mathrm{~ns}$ of the equilibrium simulation of GluT1, given that a transition occurs from the IF to the OF conformation within the first tens of ns (Fig. 3B) GlpT seems to have a more pronounced change in the interhelical angle between $\mathrm{H} 1$ and $\mathrm{H} 7$. We note that after equilibration, the $\langle\mathrm{H} 1, \mathrm{H} 7\rangle$ angle increases again to some extent in GkPOT simulations. This is potentially due to the fact that the change imposed on the $\langle\mathrm{H} 1, \mathrm{H} 7\rangle$ angle based on the LacY structure (see Theoretical Methods) is more than the actual change in $\langle\mathrm{H} 1, \mathrm{H} 7\rangle$ during thie IF-OF transition. The outcome after the equiliubration, is more closely similar to that of GluT1, which is less than 10 degrees. On the oher hand, the $\langle\mathrm{H} 1, \mathrm{H} 7\rangle$ change in GlpT is about 20 degrees. For $\langle\mathrm{H} 5, \mathrm{H} 11\rangle$, GluT1 is the transporter with the highest change. All three proteins, however, show a significant correlation between the changes in $\langle\mathrm{H} 1, \mathrm{H} 7\rangle$ and $\langle\mathrm{H} 5, \mathrm{H} 11\rangle$, in line with the rocker-switch mechanism. 


\section{Local Conformational Changes}

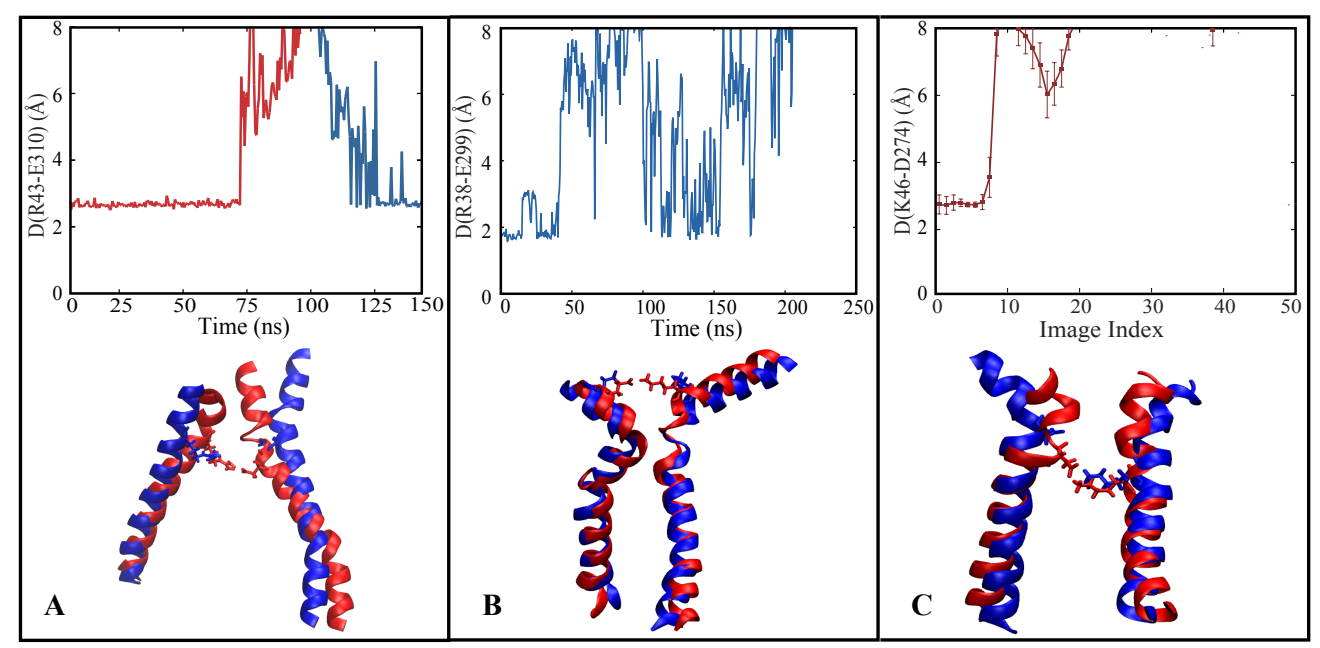

Figure 4: Minimum donor-acceptor distance measurements between the key inter-bundle salt-bridge forming amino acids (A) R43 and E310 in GkPOT, (B) R38 and E299 in GluT1, and (C) K46 and D274 in GlpT.

As GkPOT transitions toward the OF state, an important inter-bundle salt bridge between R43 in H1 and E310 in H7 becomes disrupted, and appears to break after 75 ns of the SMD pulling (Fig. 4A). The salt bridge forms again after the protein reaches the equilibrated OF conformation, although the strength of this salt bridge is extremely weakened (see the salt bridge dynamics during follow-up equilibriun simulations in Supporting Information).

The GluT1 extracelluar gating is directly influenced by the interactions between TM helices H1 and H7. This is consistent with both GkPOT and GlpT (Figure 3). However, unlike GkPOT and GlpT, the interactions between $\mathrm{H} 1$ and $\mathrm{H} 7$ take place on the extracelluar side of the protein as opposed to being in transmembrane region (Fig. 4B). The salt bridges observed in the TM region of both GkPOT and GlpT are necessary for protein stability and play a key role in substrate translocation (Fig. 4). A salt bridge between Arg 38 in H1 and Glu 299 in H7 is observed in the very beginning of the GluT1 simulation, as it remains in the IF. To allow GluT1 to move into the OF conformation, the ICH provides the cytoplasmic gating by moving into a position to interact with both H5 and H11. By forming two very strong salt bridges with H5 and H11 (Glu 243 and Arg 153, Glu 247 and Arg 400) 
(Supplementary Information), this allows for the ICH to remain locked into position . From this stability we observe the interhelical angles between H5-H11 to exhibit the same angular change of of about 15-20 degrees during the transitions between IF and OF conformations.

GlpT, on the other had, has the strongest correlation between the local events (i.e., salt bridge formation/disruption) and global conformational changes (interheclical angle changes). The interbundle salt bridges seem to play an important role in the transport mechanism of MFS transporters; however, the exact location and the exact function of these salt bridgest make it quire difficult to draw any conclusion on this issue. The interbundle salt bridges seem to stabilize the IF conformation in all three proteins and the IF-OF transition seems to require the disuption of this salt bridge. The OF state, however, may or may not be associated with the presence or absence of thi salt bridge.

\section{Water Accessibility at Cytoplasmic and Peripliasmic Gates}

Figure 5 shows the transition from the IF to OF conformations monitored with the water profile along the pore and the average water count at the cytoplasmic and periplasmic gates. The average water count in the pore extenuates the pronunciation of the OF conformation that is present from the pulling simulation (Figure 4 C-E). GlpT and GluT1 both display similar water profiles (Supporting Information). 

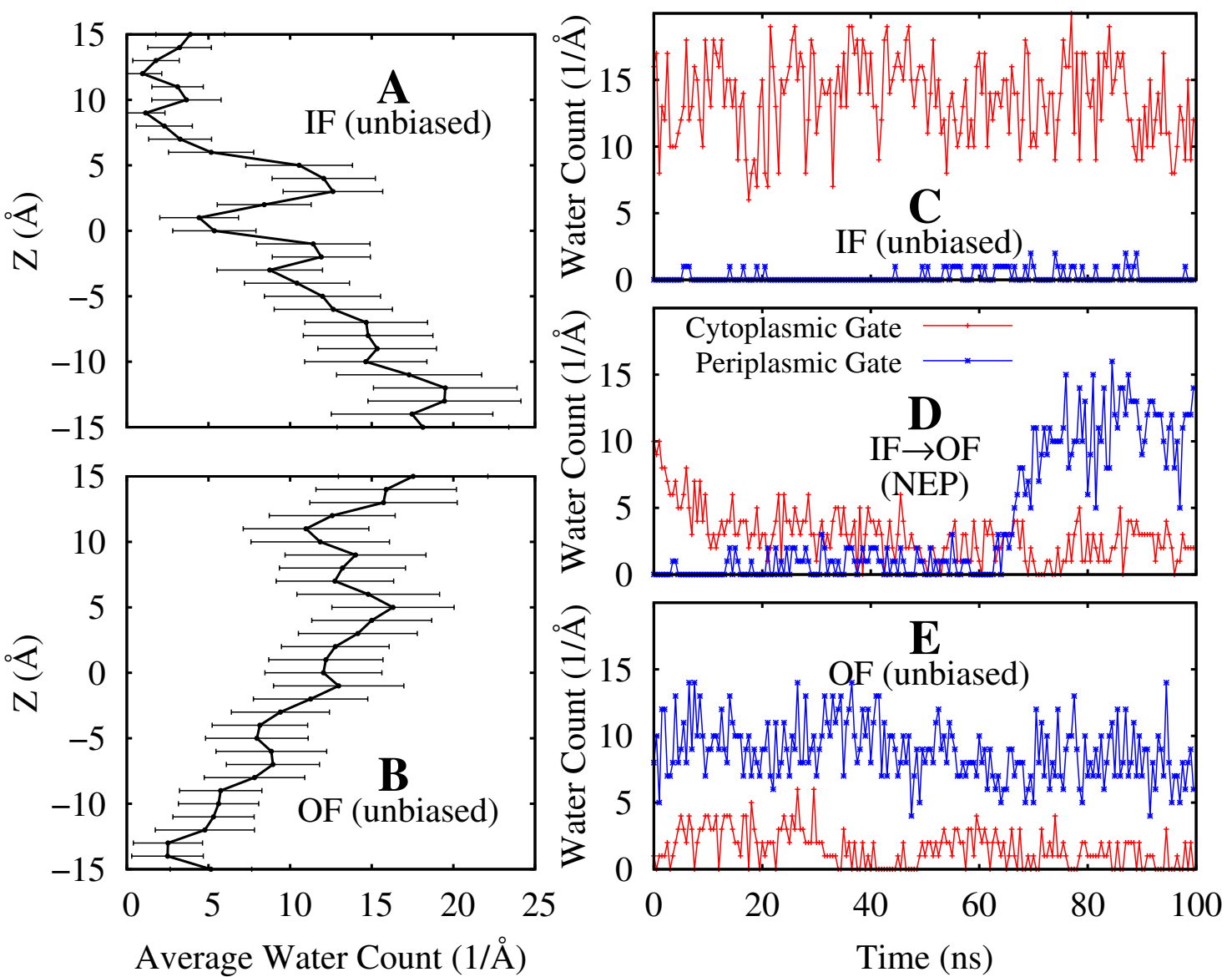

Figure 5: GkPOT water accessibility along the pore (A,B) and at peri- (blue) and cytoplasmic (red) gates during different stages of equilibrium and nonequilibrium simulations: (C) $100 \mathrm{~ns}$ of equilibrium simulation of GkPOT in the IF state prior to SMD (D) $100 \mathrm{~ns}$ of SMD as described before for GkPOT, and (E) 100 ns of follow-up unbiased MD after transitioning to the OF state. The water profiles and the error bars in $(\mathrm{A})$ and $(\mathrm{B})$ are generated from the same simulations used for panels $(\mathrm{C})$ and $(\mathrm{E})$. 


\section{Conclusion}

Employing equilibrium and nonequilibrium all-atom MD simulations of GkPOT, GluT1, and GlpT, we have demonstrated a thorough comparison of the conformational dynamics of the three different classes of MFS transporters; i.e., symporters, uniporters, and antiporters. In this study, we only focused on the apo protein. The three systems are quite different in terms of the timescale of the IF-OF transition; however, we observe various similarities between the three systems including the involvement of $\mathrm{H} 1 / \mathrm{H} 7$ helices in periplasmic/extracellular gating, the involvement of $\mathrm{H} 5 / \mathrm{H} 11$ helices in cytoplasmic gating, and the interbundle salt bridge formation in stabilizing the IF state. There are notable differences between these three systems including: (1) the smaller change of $\langle\mathrm{H} 1, \mathrm{H} 7\rangle$ angle in GkPOT and GluT1 as compared to GlpT, (2) the larger change of $\langle\mathrm{H} 5, \mathrm{H} 11\rangle$ angle in GluT1 as compared to GkPOT and GlpT, and (3) the formation of the interbundle salt bridge in the OF state for the GkPOT protein as compated to GluT1 and GlpT.

The presented study provides a detailed picture of the similarities and differences associated with the IF $\leftrightarrow \mathrm{OF}$ conformational transition of three transporters from three distinct classes of MFS superfamily. If combined with similar simulations in the presence of physiologically relevant substrates and cotransported species, the reported simulations could provide a full description of transport cycles of MFS transporters.

\section{Acknowledgement}

This research is supported by the University of Arkansas, Fayetteville. This research is part of the Blue Waters sustained-petascale computing project, which is supported by the National Science Foundation (awards OCI-0725070 and ACI-1238993) and the state of Illinois. Blue Waters is a joint effort of the University of Illinois at Urbana-Champaign and its National Center for Supercomputing Applications. This work also used the Extreme Science 
and Engineering Discovery Environment (allocation MCB150129), which is supported by

National Science Foundation grant number ACI-1548562. This research is also supported by the Arkansas High Performance Computing Center which is funded through multiple National Science Foundation grants and the Arkansas Economic Development Commission.

\section{References}

(1) Reddy, V. S.; Shlykov, M. A.; Castillo, R.; Sun, E. I.; Saier, M. H. The major facilitator superfamily (MFS) revisited. FEBS J. 2012, 279, 2022-2035.

(2) Pao, S. S.; Paulsen, I. T.; Saier, M. H., Jr. Major Facilitator Superfamily. Microbiol. Mol. Biol. Rev. 1998, 62, 1-34.

(3) Abramson, J.; Smirnova, I.; Kasho, V.; Verner, G.; Kaback, H. R.; Iwata, S. Structure and Mechanism of the Lactose Permease of Escherichia coli. Science 2003, 301, 610615.

(4) Guan, L.; Hu, Y.; Kaback, H. R. Aromatic Stacking in the Sugar Binding site of Lactose Permease. Biochemistry 2003, 42, 1377-1382.

(5) Guan, L.; Kaback, H. Lessons from Lactose Permease. Annu. Rev. Biophys. Biomol. Struct. 2006, 35, 67-91.

(6) Lawrence, M. G.; Lindahl, L.; Zengel, J. M. Effects on translation pausing of alterations in protein and RNA components of the ribosome exit tunnel. J. Bacteriol. 2008, 190, $5862-5869$.

(7) Quistgaard, E. M.; Löw, C.; Moberg, P.; Trésaugues, L.; Nordlund, P. Structural basis for substrate transport in GLUT-homology family of monosaccharide transporters. Nat. Struct. Mol. Biol. 2013, 20, 766-768. 
(8) Yin, Y.; Jensen, M. Ø.; Tajkhorshid, E.; Schulten, K. Sugar Binding and Protein Conformational Changes in Lactose Permease. Biophys. J. 2006, 91, 3972-3985.

(9) Yan, N. Structural advances for the major facilitator superfamily (MFS) transporters. Trends in Biochemical Sciences 2013, 38, 151-159.

(10) Wood, I. S.; Trayhurn, P. Glucose transporters (GLUT and SGLT): expanded families of sugar transport proteins. British Journal of Nutrition 2003, 89, 3-9.

(11) Solcan, N.; Kwok, J.; Fowler, P. W.; Cameron, A. D.; Drew, D.; Iwata, S.; Newstead, S. Alternating access mechanism in the POT family of oligopeptide transporters. EMBO J. 2012, 31, 3411-3421.

(12) Guettou, F.; Quistgaard, E. M.; Tresaugues, L.; Moberg, P.; ; Jorgerschold, C.; Jong, A. J.; Nordlund, P.; Loew, C. Structural insights into substrate recognition in proton-dependent oligopeptide transporters. EMBO Rep. 2013, 14, 804-810.

(13) Thorens, B.; Mueckler, M. Glucose transporters in the 21st Century. Am. J. Physiol. - Endoc. M. 2010, 298, E141-E145.

(14) Deng, D.; Xu, C.; Sun, P.; Wu, J.; Yan, C.; Hu, M.; Yan, N. Crystal structure of the human glucose transporter GLUT1. Nature 2014, 510, 121-125.

(15) Yan, N. Structural advances for the major facilitator superfamily (MFS) transporters. Trends Biochem. Sci. 2013, 38, 151-159.

(16) Doki, S.; Kato, H. E.; Solcan, N.; Iwaki, M.; Koyama, M.; Hattori, M.; Iwase, N.; Tsukazaki, T.; Sugita, Y.; Kandori, H. et al. Structural basis for dynamic mechanism of proton-coupled symport by the peptide transporter POT. Proc. Natl. Acad. Sci. USA 2013, 110, 11343-11348.

(17) Yang, Q.; Ma, Y.; Zhao, Y.; She, Z.; Wang, L.; Li, J.; Wang, C.; Deng, Y. Accelerated drug release and clearance of PEGylated epirubicin liposomes following repeated injec- 
tions: a new challenge for sequential low-dose chemotherapy. International Journal of Nanomedicine 2013, 8, 1257 - 1268.

(18) Lyons, J. A.; Parker, J. L.; Solcan, N.; Brinth, A.; Li, D.; Shah, S. T.; Caffrey, M.; Newstead, S. Structural basis for polyspecificity in the POT family of proton-coupled oligopeptide transporters. EMBO Rep. 2014, 15, 886 - 893.

(19) Newstead, S.; Drew, D.; Cameron, A. D.; Postis, V. L.; Xia, X.; Fowler, P. W.; Ingram, J. C.; Carpenter, E. P.; Sansom, M. S.; McPherson, M. J. et al. Crystal structure of a prokaryotic homologue of the mammalian oligopeptide-proton symporters, PepT1 and PepT2. EMBO J. 2011, 30, 417-426.

(20) Quistgaard, E. M.; Martinez Molledo, M.; Lw, C. Structure determination of a major facilitator peptide transporter: Inward facing PepTSt from Streptococcus thermophilus crystallized in space group P3121. PLOS ONE 2017, 12, 1-20.

(21) Deng, D.; Sun, P.; Yan, C.; Ke, M.; Jiang, X.; Xiong, L.; Ren, W.; Hirata, K.; Yamamoto, M.; Fan, S. et al. Molecular basis of ligand recognition and transport by glucose transporters. Nature 2015, 526, 391.

(22) Yan, N. A glimpse of membrane transport through structuresadvances in the structural biology of the GLUT glucose transporters. Journal of molecular biology 2017, 429, $2710-2725$.

(23) Elvin, C. M.; Hardy, C. M.; Rosenberg, H. Pi exchange mediated by the GlpTdependent sn-glycerol-3-phosphate transport system in Escherichia coli. Journal of Bacteriology 1985, 161, $1054 \mathrm{LP}-1058$.

(24) Law, C. J.; Almqvist, J.; Bernstein, A.; Goetz, R. M.; Huang, Y.; Soudant, C.; Laaksonen, A.; Hovmöller, S.; Wang, D.-N. Salt-bridge Dynamics Control Substrate-induced Conformational Change in the Membrane Transporter GlpT. Journal of Molecular Biology 2008, 378, 828-839. 
(25) Huang, Y.; Lemieux, M. J.; Song, J.; Auer, M.; Wang, D.-N. Structure and Mechanism of the Glycerol-3-Phosphate Transporter from $<\mathrm{em}>$ Escherichia coli $</ \mathrm{em}>$. Science 2003, 301, $616 \mathrm{LP}-620$.

(26) Immadisetty, K.; Hettige, J.; Moradi, M. What Can and Cannot Be Learned from Molecular Dynamics Simulations of Bacterial Proton-Coupled Oligopeptide Transporter GkPOT? The Journal of Physical Chemistry B 2017, 121, 3644-3656.

(27) Galochkina, T.; Ng Fuk Chong, M.; Challali, L.; Abbar, S.; Etchebest, C. New insights into GluT1 mechanics during glucose transfer. Scientific Reports 2019, 9, 998.

(28) Moradi, M.; Enkavi, G.; Tajkhorshid, E. Atomic-level characterization of transport cycle thermodynamics in the glycerol-3-phosphate:phosphate antiporter. Nature Communications 2015, 6, 8393.

(29) Isralewitz, B.; Stone, J.; Schulten, K. Timeline: an interactive raster plot to identify events in molecular dynamics trajectories. 2011, Submitted.

(30) Schlick, T.; Li, B.; Olson, W. The influence of salt on the structure and energetics of supercoiled DNA. Biophys. J. 1994, 67, 2146-2166.

(31) Moradi, M.; Tajkhorshid, E. Mechanistic picture for conformational transition of a membrane transporter at atomic resolution. Proc. Natl. Acad. Sci. USA 2013, 110, $18916-18921$.

(32) Moradi, M.; Tajkhorshid, E. Computational Recipe for Efficient Description of LargeScale Conformational Changes in Biomolecular Systems. Journal of Chemical Theory and Computation 2014, 10, 2866-2880.

(33) Fakharzadeh, A.; Moradi, M. Effective Riemannian Diffusion Model for Conformational Dynamics of Biomolecular Systems. J. Phys. Chem. Lett. 2016, 7, 4980-4987. 
(34) Jo, S.; Kim, T.; Im, W. Automated builder and database of protein/membrane complexes for molecular dynamics simulations. PLoS One 2007, 2, e880.

(35) Lee, J.; Cheng, X.; Swails, J. M.; Yeom, M. S.; Eastman, P. K.; Lemkul, J. A.; Wei, S.; Buckner, J.; Jeong, J. C.; Qi, Y. et al. CHARMM-GUI input generator for NAMD, GROMACS, AMBER, OpenMM, and CHARMM/OpenMM simulations using the CHARMM36 additive force field. J. Chem. Theory Comp. 2016, 12, 405-413.

(36) Jorgensen, W. L.; Chandrasekhar, J.; Madura, J. D.; Impey, R. W.; Klein, M. L. Comparison of simple potential functions for simulating liquid water. J. Chem. Phys. 1983, 79, 926-935.

(37) Klauda, J. B.; Venable, R. M.; Freites, J. A.; O'Connor, J. W.; Tobias, D. J.; Mondragon-Ramirez, C.; Vorobyov, I.; MacKerell Jr., A. D.; Pastor, R. W. Update of the CHARMM all-atom additive force field for lipids: Validation on six lipid types. J. Phys. Chem. B 2010, 114, 7830-7843.

(38) Best, R. B.; Zhu, X.; Shim, J.; Lopes, P. E. M.; Mittal, J.; Feig, M.; MacKerell, A. D. Optimization of the additive CHARMM all-atom protein force field targeting improved sampling of the backbone $\phi, \psi$ and side-chain $\chi 1$ and $\chi 2$ dihedral angles. J. Chem. Theory Comp. 2012, 8, 3257-3273.

(39) Phillips, J. C.; Braun, R.; Wang, W.; Gumbart, J.; Tajkhorshid, E.; Villa, E.; Chipot, C.; Skeel, R. D.; Kale, L.; Schulten, K. Scalable molecular dynamics with NAMD. J. Comp. Chem. 2005, 26, 1781-1802.

(40) Reid, J. K. In Large Sparse Sets of Linear Equations; Reid, J. K., Ed.; Academic Press: London, 1971; pp 231-254.

(41) Martyna, G. J.; Tobias, D. J.; Klein, M. L. Constant pressure molecular dynamics algorithms. J. Chem. Phys. 1994, 101, 4177-4189. 
(42) Feller, S. E.; Zhang, Y.; Pastor, R. W.; Brooks, B. R. Constant pressure molecular dynamics simulation: The Langevin piston method. J. Chem. Phys. 1995, 103, 46134621.

(43) Darden, T.; York, D.; Pedersen, L. G. Particle mesh Ewald: An $N \cdot \log (N)$ method for Ewald sums in large systems. J. Chem. Phys. 1993, 98, 10089-10092.

(44) Dang, S.; Sun, L.; Huang, Y.; Lu, F.; Liu, Y.; Gong, H.; Wang, J.; Yan, N. Structure of a fucose transporter in an outward-open conformation. Nature 2010, 467, 734-738.

(45) Humphrey, W.; Dalke, A.; Schulten, K. VMD: visual molecular dynamics. J. Mol. Graphics 1996, 14, 33-38. 